\title{
Signal Reconstruction in Multidimensional Sensor Fields
}

\author{
Alessandro Nordio and Carla-Fabiana Chiasserini \\ Politecnico di Torino,Italy \\ Email: \{nordio,chiasserini\}@polito.it
}

\author{
Emanuele Viterbo \\ DEIS, Università della Calabria, Italy \\ Email: viterbo@deis.unical.it
}

\begin{abstract}
Sensor networks are often used for environmental monitoring. In this context, sampling and reconstruction of a physical field is a fundamental issue to solve. We consider a bandlimited, multidimensional field and study the quality level of its reconstruction when the sensor measurements are noisy and the number of available sensors varies. We find that, for an exact analysis of the problem, we would need the closed-form expression of the eigenvalue distribution of the reconstruction matrix, which, to the best of our knowledge, is still unknown. Thus, we first derive a closed-form expression of the distribution moments, and we find that the eigenvalue distribution tends to the Marčenko-Pastur distribution as the field dimension goes to infinity. We then apply our findings to the study of the MSE of the reconstructed field, when linear reconstruction techniques are used, and we derive an approximation that is very tight already for a 3-dimensional field.
\end{abstract}

\section{INTRODUCTION}

One of the most popular applications of wireless sensor networks is environmental monitoring. In general, a physical phenomenon (hereinafter called signal or field) may vary over both space and time, with some band limitation in both domains. In this work, we address the problem of sampling and reconstruction of a bandlimited, $d$-dimensional sensor field, where $d$ may take into account spatial as well as temporal dimensions. We assume that sensors are randomly deployed over a geographical area to sample the phenomenon of interest, and that are transferred from the sensors to a common datacollecting unit, the so-called sink node.

The issue we deal with is the field reconstruction from a collection of samples that, being the sensors randomly deployed, are not uniformly spaced. Let us assume that the sampling points are known at the sink node, because (i) either sensors are located at pre-defined positions or their position can be estimated through a localization technique, and (ii) the sampling time is either periodic or included in the information sent to the sink. Here, we do not deal with issues related to information transport, and consider that all data are correctly received at the sink node. Furthermore, we consider that the field samples are corrupted by additive noise, due to quantization, round-off errors or quality of the sensing device.

Several efficient and fast algorithms have been proposed to numerically reconstruct or approximate a signal in these cases, which amount to the solution of a linear system [1]. A widely used technique consists in processing the sensor measures by means of a linear filter, which is a function of the system parameters known at the sink. In [2], we have found that, to study the quality of linear reconstruction, we need the eigenvalue distribution of the reconstruction matrix. However, obtaining such a distribution is still an open problem. In this work, we first extend the system model and the problem formulation presented in [2] to the case of multidimensional fields (Section II). Then, we derive a closed-form expression of the moments of the eigenvalue distribution (Section III), through asymptotic analysis. By using the moments expressions, we prove that the eigenvalue distribution of the matrix representing the sampling system tends to the Marčenko-Pastur distribution as the field dimension $d \rightarrow \infty$ (Section IV). We apply our results to the study of the mean square error (MSE) of the field estimate, when the reconstruction at the sink is performed through linear filtering and the sensor measurements are affected by noise. We show that, by using the Marčenko-Pastur distribution instead of the actual eigenvalue distribution, we obtain an approximation to the MSE of the reconstructed field, which is very tight already for a 3-dimensional field (Section V).

\section{SYSTEM MODEL}

Here, we present the multidimensional formulation of our reconstruction problem. Also, we give some background on linear reconstruction techniques and generalize to the multidimensional case some results on the MSE of the reconstructed field.

\section{A. Irregular sampling of bandlimited signals}

Let us consider a $d$-dimensional field model $(d \geq 1)$, where $r$ sensors are located in the hypercube $\mathcal{H}=\left\{\mathbf{x} \mid \mathbf{x} \in[0,1)^{d}\right\}$ and measure the value of a bandlimited signal $s(\mathbf{x})^{1}$. We suppose that sensors can represent each sample with a sufficient number of bits so that the quantization error is negligible. Furthermore, we assume that the sensor sampling points are known. At first, we consider that they are deterministic, then we will assume that they are i.i.d. random variables uniformly distributed in the hypercube $\mathcal{H}$.

Let $\mathcal{X}=\left\{\mathbf{x}_{1}, \ldots, \mathbf{x}_{r}\right\}$, with $\mathbf{x}_{q}=\left[x_{q, 1}, \ldots, x_{q, d}\right]^{\mathrm{T}} \in \mathcal{H}$, $q=1, \ldots, r$, be the set of sampling points, and $\mathbf{s}=$ $\left[s_{1}, \ldots, s_{r}\right]^{\mathrm{T}}, s_{q}=s\left(\mathbf{x}_{q}\right)$ the values of the corresponding

${ }^{1}$ Lower case bold letters denote column vectors, while upper case bold letters denote matrices. The $(h, k)$-th entry of a matrix $\mathbf{X}$ is denoted by $(\mathbf{X})_{h, k}$, and the sign $(\cdot)^{\mathrm{T}}$ represents the transposition operator. $\mathbb{E}[\cdot]$ is the average operator, while the identity matrix is denoted by $\mathbf{I}$. The superscript $(\cdot)^{\dagger}$ denotes the conjugate transposition operator 
field samples. We represent the spectrum of $s(\mathbf{x})$ by using $2 M+1$ harmonics per dimension ${ }^{2}$, i.e., through a random column vector $\mathbf{a}=\left[a_{-N}, \ldots, a_{+N}\right]^{\mathrm{T}}$ of size $(2 M+1)^{d}$, where $N=\frac{(2 M+1)^{d}-1}{2}$, and we assume $\mathbb{E}\left[\mathbf{a a}^{\dagger}\right]=\sigma_{a}^{2} \mathbf{I}$. Then, a strictly bandlimited signal over $\mathcal{H}$ can be written in terms of Fourier series as the weighted sum of $(2 M+1)^{d}$ harmonics:

$$
s(\mathbf{x})=\frac{1}{\sqrt{(2 M+1)^{d}}} \sum_{\ell} a_{\nu(\ell)} \mathrm{e}^{2 \pi \mathbf{i x}^{\mathrm{T}} \boldsymbol{\ell}}
$$

where $\ell=\left[\ell_{1}, \cdots, \ell_{d}\right]^{\mathrm{T}}, \ell_{m}=-M, \cdots, M$ is a vector of integers, $\sum_{\ell}$ represents a $d$-dimensional sum, and the function

$$
\nu(\ell)=\sum_{m=1}^{d}(2 M+1)^{m-1} \ell_{m}
$$

$-N \leq \nu(\ell) \leq+N$, maps the vector $\ell$ onto a scalar index. Following [1], we write the vector of field values $\mathbf{s}$ as a function of the spectrum: $\mathbf{s}=\mathbf{G}_{d}^{\dagger} \mathbf{a}$, where $\mathbf{G}_{d}$ is the $(2 M+1)^{d} \times r$ Fourier matrix:

$$
\left(\mathbf{G}_{d}\right)_{\nu(\ell), q}=\frac{1}{\sqrt{(2 M+1)^{d}}} \mathrm{e}^{-2 \pi \mathrm{i} \mathbf{x}_{q}^{\mathrm{T}} \boldsymbol{\ell}}
$$

If the sensor measures of the field, $\mathbf{p}=\left[p_{1}, \ldots, p_{r}\right]^{\mathrm{T}}$, are affected by noise, then the relation between sensor samples and field spectrum can be written as:

$$
\mathbf{p}=\mathbf{s}+\mathbf{n}=\mathbf{G}_{d}^{\dagger} \mathbf{a}+\mathbf{n}
$$

where the noise is represented by the $r$-size, zero-mean random vector $\mathbf{n}$, with covariance matrix $\mathbb{E}\left[\mathbf{n n}^{\dagger}\right]=\sigma_{n}^{2} \mathbf{I}_{2 M+1}$. We define the signal-to-noise ratio on the measure as:

$$
\mathrm{SNR}_{m} \triangleq \frac{\sigma_{a}^{2}}{\sigma_{n}^{2}} \triangleq \frac{1}{\alpha}
$$

\section{B. Sampling rate}

Following [2], we introduce the parameter $\beta$ defined as:

$$
\beta=\frac{(2 M+1)^{d}}{r}
$$

This value represents the ratio between the number of harmonics used for the field reconstruction and the number of sensors sampling the field. In the case of regular sampling perfect reconstruction is achieved for $\beta \in[0,1)$ and, for $\beta=1$, we have the limit fixed by the Nyquist theorem for the perfect recovery of the signal. In the following we consider $\beta \in[0,1)$. Note also that $1 / \beta$ represents the oversampling factor with respect to the Nyquist sampling rate.

\section{Some results on reconstruction quality}

Given an estimate $\hat{\mathbf{a}}$ of the field spectrum a, the reconstructed signal is:

$$
\hat{s}(\mathbf{x})=\frac{1}{\sqrt{(2 M+1)^{d}}} \sum_{\ell} \hat{a}_{\nu(\ell)} \mathrm{e}^{2 \pi \mathrm{i} \mathbf{x}^{\mathrm{T}} \boldsymbol{\ell}}
$$

${ }^{2}$ Without loss of generality, $2 M+1$ represents the maximum number of harmonics among all spatial and temporal dimensions
As a reconstruction performance metric we consider the MSE of the field estimate, which, for any given set of sampling points $\mathcal{X}$, is defined as:

$$
\begin{aligned}
\operatorname{MSE}_{\mathcal{X}} & =\underset{\mathbf{a}, \mathbf{n}}{\mathbb{E}}\left[\int_{\mathcal{H}}|\hat{s}(\mathbf{x})-s(\mathbf{x})|^{2} \mathrm{~d} \mathbf{x}\right] \\
& =\frac{1}{(2 M+1)^{d}} \underset{\mathbf{a}, \mathbf{n}}{\mathbb{E}}\left[\|\hat{\mathbf{a}}-\mathbf{a}\|^{2}\right]
\end{aligned}
$$

where the operator $\mathbb{E}[\cdot]$ averages with respect to the subscripted random vectors. Note that (6) still assumes that the sampling points are deterministic; later in the paper, this assumption will be removed.

For linear models such as (3) we estimate the field spectrum by using a linear filter, $\mathbf{B}$, such that $\hat{\mathbf{a}}=\mathbf{B}^{\dagger} \mathbf{p}$ where $\mathbf{B}$ is an $r \times(2 M+1)^{d}$ matrix. In particular, we consider the linear filter providing the best performance in terms of MSE, that is, the linear minimum MSE (LMMSE) filter, thus:

$$
\mathbf{B}=\mathbf{G}_{d}^{\dagger}\left(\mathbf{R}_{d}+\alpha \mathbf{I}\right)^{-1}
$$

where $\mathbf{R}_{d}=\mathbf{G}_{d} \mathbf{G}_{d}^{\dagger}$. Notice that this reconstruction technique does not require interpolation and can be viewed as a generalization of the classical discrete Fourier transform (DFT) to irregularly sampled signals.

In [2], we showed that a simple and effective tool to evaluate the performance of large finite systems is asymptotic analysis. We computed the MSE by letting the field number of harmonics and the number of samples grow to infinity, while their ratio $\beta=(2 M+1)^{d} / r$ is kept constant. We observed the validity of asymptotic analysis results, even for small values of $M$ and $r$. Similarly, here we consider as performance metric the asymptotic average MSE, normalized to $\sigma_{a}^{2}$ :

$$
\mathrm{MSE}_{\infty}=\lim _{M, r \rightarrow+\infty} \frac{1}{\sigma_{a}^{2}} \underset{\mathcal{X}}{\mathbb{E}}\left[\operatorname{MSE}_{\mathcal{X}}\right]=\underset{\lambda_{d, \beta}}{\mathbb{E}}\left[\frac{\alpha \beta}{\lambda_{d, \beta}+\alpha \beta}\right]
$$

where $\lambda_{d, \beta}$ is a random variable with probability density function (pdf) $f_{d, \beta}(x)$, distributed as the asymptotic eigenvalues of $\mathbf{T}_{d}=\beta \mathbf{R}_{d}=\beta \mathbf{G}_{d} \mathbf{G}_{d}^{\dagger}$. In (8), we consider that the sampling points are randomly distributed in the hypercube $\mathcal{H}$, and the average is over all possible realizations of the set $\mathcal{X}$. In particular, from now on, we carry out our analysis under the assumption that the elements of the set $\mathcal{X}$ are independent random vectors, with i.i.d. entries, uniformly distributed in the hypercube $\mathcal{H}$. The subscripts $d$ and $\beta$ of $\lambda$ indicate that the distribution of the asymptotic eigenvalues of $\mathbf{T}_{d}$ depends on both the field dimension $d$ and the parameter $\beta$.

The $(2 M+1)^{d} \times(2 M+1)^{d}$ Hermitian Toeplitz matrix $\mathbf{T}_{d}$ plays an important role in our analysis. Its entries are given by

$$
\left(\mathbf{T}_{d}\right)_{\nu(\ell), \nu\left(\boldsymbol{\ell}^{\prime}\right)}=\frac{1}{r} \sum_{q=1}^{r} \mathrm{e}^{2 \pi \mathrm{i} \mathbf{x}_{\mathrm{q}}^{\mathrm{T}}\left(\boldsymbol{\ell}-\ell^{\prime}\right)}
$$

where $\ell, \ell^{\prime} \in[-M, \ldots, M]^{d}$. A detailed definition of $\mathbf{T}_{2}$ can be found in [4]. 


\section{ClOSED-FORM EXPRESSION OF THE MOMENTS OF THE ASYMPTOTIC EIGENVALUE PDF}

Let $\lambda_{d, \beta}$ be the asymptotic eigenvalue of $\mathbf{T}_{d}$, in the $d$ dimensional case and for a given $\beta$. Looking at the asymptotic expression of the LMMSE in (8), we note that a closed-form expression of the eigenvalue distribution of the reconstruction matrix, $f_{d, \beta}(x)$, would be required for its analytical evaluation. Unfortunately, this calculation seems to be prohibitive, thus, as a first step, we derive a closed-form expression of the moments of $\lambda_{d, \beta}$, i.e., $\mathbb{E}\left[\lambda_{d, \beta}^{p}\right]$, for any $d, \beta$, and positive integer $p$.

In the limit for $M$ and $r$ growing to infinity with constant $\beta$, the expression of $\mathbb{E}\left[\lambda_{d, \beta}^{p}\right]$ can be easily obtained from the powers of $\mathbf{T}_{d}$ as

$$
\mathbb{E}\left[\lambda_{d, \beta}^{p}\right]=\lim _{\substack{M, r \rightarrow+\infty \\ \frac{(2 M+1)^{d}}{r}=\beta}} \frac{1}{(2 M+1)^{d}} \operatorname{Tr}\left\{\underset{\mathcal{X}}{\mathbb{E}}\left[\mathbf{T}_{d}^{p}\right]\right\}
$$

From (10) and using (9), the expression of the $p$-th moment of $\lambda_{d, \beta}$ can be written as:

$$
\begin{aligned}
\mathbb{E}\left[\lambda_{d, \beta}^{p}\right]= & \lim _{\substack{r \rightarrow+\infty \\
r^{p}(2 M+1)^{d}}} \sum_{\mathbf{q} \in \mathcal{Q}} \sum_{\mathbf{L} \in \mathcal{L}_{d}} . \\
& \underset{\mathcal{X}}{\mathbb{E}}\left[\exp \left(2 \pi \mathrm{i} \sum_{i=1}^{p} \mathbf{x}_{q_{i}}^{\mathrm{T}}\left(\boldsymbol{\ell}_{i}-\boldsymbol{\ell}_{[i+1]}\right)\right)\right]
\end{aligned}
$$

where $\mathcal{Q}=\left\{\mathbf{q} \mid \mathbf{q}=\left[q_{1}, \ldots, q_{p}\right]^{\mathrm{T}}, \quad q_{i}=1, \ldots, r\right\}$ and $\mathcal{L}_{d}=\left\{\mathbf{L} \mid \mathbf{L}=\left[\ell_{1}, \ldots, \ell_{p}\right]\right\}, \ell_{i}=\left[\ell_{i_{1}}, \cdots \ell_{i_{d}}\right]^{\mathrm{T}}, \ell_{i_{m}}=$ $-M, \cdots, M$ and where $[i+1]=i+1$ if $1 \leq i<p$, and $[i+1]=1$ if $i=p$. The average $\underset{\mathcal{X}}{\mathbb{E}}[\cdot]$ is performed over the set of independent random vectors $\mathcal{X}=\left\{\mathbf{x}_{1}, \ldots, \mathbf{x}_{r}\right\}$ with independent entries uniformly distributed in $[0,1)$.

Now, to obtain a closed-form expression of the distribution moments, we rewrite (11) by using set partitioning. Let $\mathcal{P}=$ $\{1, \ldots, p\}$ be the set of integers from 1 to $p$. We observe that any given vector $\mathbf{q} \in \mathcal{Q}$ partitions the set $\mathcal{P}$ into $1 \leq k(\mathbf{q}) \leq p$ disjoint non-empty subsets $\mathcal{P}_{1}(\mathbf{q}), \ldots, \mathcal{P}_{k}(\mathbf{q})$ where $\mathcal{P}_{j}, j=$ $1, \ldots, k(\mathbf{q})$, is the set of indices of the entries of $\mathbf{q}$ taking the same value $\gamma_{j}$. i.e., $\mathcal{P}_{j}(\mathbf{q})=\left\{i \in \mathcal{P} \mid q_{i}=\gamma_{j}\right\}$, and $k(\mathbf{q})$ is the number of distinct values $\gamma_{j}$ taken by the entries of vector q.

To represent the partitions of $\mathcal{P}$, we build a tree of depth $p$ as follows (see Figure 1). A label from the set $\mathcal{P}=\{1, \ldots, p\}$ is given to each node of the tree, starting from the root which is labeled by 1 . Each node generates $m+1$ leaves, labeled in increasing order from 1 to $m+1$, where $m$ is the largest label in the path from the root to such node. Note that, at level $p$, any value in $\{1, \ldots, p\}$ is used to label the leaves at least once. Then, given a tree of depth $p$, we define $\boldsymbol{\omega}=\left[\omega_{1}, \ldots, \omega_{p}\right]$ as a path of length $p$ from the tree's root to a leaf. Also, let $k(\boldsymbol{\omega})$ be the number of distinct labels in $\boldsymbol{\omega}$. We observe that any given path $\boldsymbol{\omega}$ partitions the set $\mathcal{P}$ into $1 \leq k(\boldsymbol{\omega}) \leq p$ disjoint nonempty subsets $\mathcal{P}_{1}(\boldsymbol{\omega}), \ldots, \mathcal{P}_{k}(\boldsymbol{\omega})$, where $k(\boldsymbol{\omega})=k(\mathbf{q})$ and $\mathcal{P}_{j}(\boldsymbol{\omega})(j=1, \ldots, k(\boldsymbol{\omega}))$ is the set of integers corresponding to the depths of the $j$-th label in the path, i.e.,

$$
\mathcal{P}_{j}(\boldsymbol{\omega})=\left\{i \in \mathcal{P} \mid \omega_{i}=j\right\}
$$

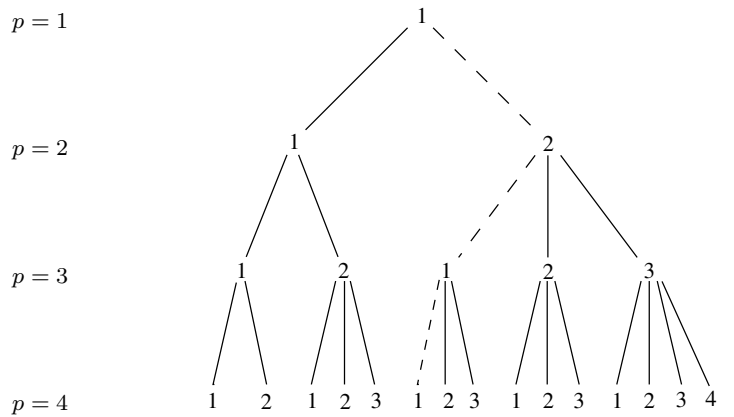

Fig. 1. Partitions tree of depth $p=4$. As an example, the path $\boldsymbol{\omega}=$ $[1,2,1,1]$ is highlighted by using dashed lines

From the discussion above, it should be clear that considering a partition of $\mathcal{P}$ is equivalent to considering a path $\boldsymbol{\omega}$ in a tree of depth $p$. Thus, after some calculations, we can rewrite (11) as

$$
\mathbb{E}\left[\lambda_{d, \beta}^{p}\right]=\sum_{k=1}^{p}\left(\sum_{\boldsymbol{\omega} \in \Omega_{p, k}} v(\boldsymbol{\omega})^{d}\right) \beta^{p-k}
$$

where $\Omega_{p}$ be the set of vectors $\boldsymbol{\omega}$, each corresponding to a distinct partition of $\mathcal{P}$, and $\Omega_{p, k} \subseteq \Omega_{p}$ is the subset of $\Omega_{p}$ containing paths with $k$ distinct labels.

It can be shown that [3], for any $\boldsymbol{\omega} \in \Omega_{p}$ (or, equivalently, a partition of $\mathcal{P}$ ) and any arbitrary integer $n$, with $n=1, \ldots, k(\boldsymbol{\omega})$, the coefficient $v(\boldsymbol{\omega})$ is given by:

$$
v(\boldsymbol{\omega})=\left.\int_{\mathbb{R}^{k(\boldsymbol{\omega})-1}} \prod_{i=1}^{p} \operatorname{sinc}\left(y_{\omega_{i}}-y_{\omega_{[i+1]}}\right)\right|_{y_{n}=0} \mathrm{~d} \mathbf{y}_{n}
$$

where $\mathbf{y}_{n}=\left[y_{1}, \ldots, y_{n-1}, y_{n+1}, \ldots, y_{k(\boldsymbol{\omega})}\right]^{\mathrm{T}} ;$ also $0 \leq$ $v(\boldsymbol{\omega}) \leq 1$. Equation (13) provides a closed-form expression of the moment $\mathbb{E}\left[\lambda_{d, \beta}^{p}\right]$, as a polynomial in $\beta$ of degree $p-1$.

As a final step, in order to compute $\mathbb{E}\left[\lambda_{d, \beta}^{p}\right]$, we need to enumerate the partitions, i.e., the vectors $\boldsymbol{\omega} \in \Omega_{p, k}$, for each $k=1, \ldots, p$. We note that $\Omega_{p}$ represents the set of partitions of a $p$-element set, thus it has cardinality $\left|\Omega_{p}\right|=B(p)$, where $B(p)$ is the $p$-th Bell number or exponential number. Furthermore, the subset $\Omega_{p, k} \subseteq \Omega_{p}$ has cardinality $S(p, k)$, which is a Stirling number of the second kind, with $B(p)=$ $\sum_{k=1}^{p} S(p, k)$.

\section{Convergence to the MarČEnko-Pastur DISTRIBUTION}

In Section III, we have shown that the moments of the asymptotic eigenvalues of $\mathbf{T}_{d}$ are polynomials in $\beta$, given by (13). In particular, the $p$-th moment $\mathbb{E}\left[\lambda_{d, \beta}^{p}\right]$ has degree $p-1$ and is given by the sum of $B(p)$ positive contributions of the form $v(\boldsymbol{\omega})^{d} \beta^{p-k(\boldsymbol{\omega})}$. Since $0<v(\boldsymbol{\omega}) \leq 1$ and $\beta>0$, for any $d$, the following inequality holds:

$$
\mathbb{E}\left[\lambda_{d+1, \beta}^{p}\right] \leq \mathbb{E}\left[\lambda_{d, \beta}^{p}\right]
$$


i.e., for any given $p$ and $\beta$, the moments of the asymptotic eigenvalues decrease as the field dimension increases. The series $\mathbb{E}\left[\lambda_{d, \beta}^{p}\right]$, as a function of $d$, is positive and monotonically decreasing, thus it converges to:

$$
\mathbb{E}\left[\lambda_{\infty, \beta}^{p}\right]=\lim _{d \rightarrow+\infty} \mathbb{E}\left[\lambda_{d, \beta}^{p}\right]
$$

It can be shown that the moments $\mathbb{E}\left[\lambda_{\infty, \beta}^{p}\right]$ are the Narayana polynomials, given by

$$
\mathbb{E}\left[\lambda_{\infty, \beta}^{p}\right]=\sum_{k=1}^{p} T(p, k) \beta^{p-k}
$$

where $T(p, k)$ are the Narayana numbers [5]. Furthermore, the random variable $\lambda_{\infty, \beta}^{p}$ follows the Marčenko-Pastur distribution with pdf:

$$
f_{\infty, \beta}(x)=\frac{\sqrt{\left(c_{1}-x\right)\left(x-c_{2}\right)}}{2 \pi x \beta}
$$

where $c_{1}, c_{2}=(1 \pm \sqrt{\beta})^{2}, 0<\beta \leq 1, c_{2} \leq x \leq c_{1}$.

Next, we apply our findings to the study of the LMMSE of a reconstructed multidimensional field; in particular, we exploit the Marčenko-Pastur distribution to compute the expectation in (8).

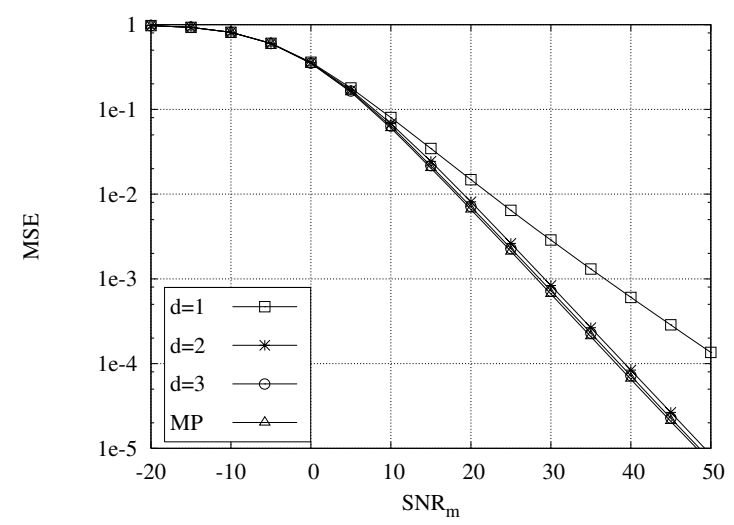

Fig. 2. MSE of the reconstructed field, for $\beta=0.4$ and $d=1,2,3$. Comparison between the MSE asymptotic value (8) and the fully analytical expression derived using the Marčenko-Pastur distribution (18)

\section{STUDY OF THE RECONSTRUCTION QUALITY THROUGH THE MARČENKO-PASTUR DISTRIBUTION}

Consider the expression in (8), which represents the MSE obtained through the LMMSE filter. By using the MarčenkoPastur distribution $f_{\infty, \beta}$ instead of $f_{d, \beta}$, we have:

$$
\operatorname{MSE}_{\infty}=\underset{\lambda_{\infty, \beta}}{\mathbb{E}}\left[\frac{\alpha \beta}{\lambda_{\infty, \beta}+\alpha \beta}\right]=\frac{2 \beta-\theta+\sqrt{\theta^{2}-4 \beta}}{2 \beta}
$$

where $\theta=1+\beta(1+\alpha)$.

Equation (18) provides an accurate approximation to the asymptotic MSE, which can be exploited to derive the quality of the reconstructed field, given a finite $d$. This is shown in Figures 2 and 3, which plot the results obtained through (8)

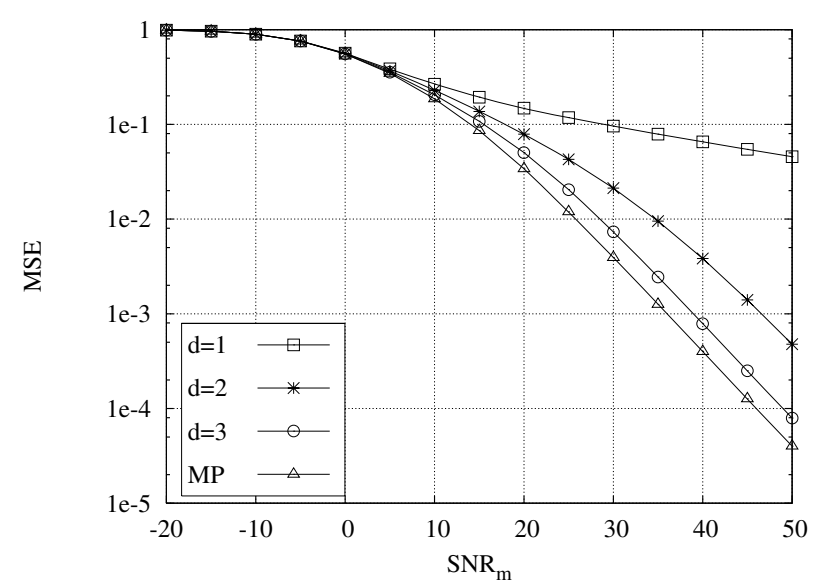

Fig. 3. MSE of the reconstructed field, for $\beta=0.8$ and $d=1,2,3$. Comparison between the MSE asymptotic value (8) and the fully analytical expression derived using the Marčenko-Pastur distribution (18)

and (18), respectively, for $\beta$ equal to 0.4 and 0.8 . We computed (8) by averaging over the eigenvalues of 200 realizations of the matrix $\mathbf{T}_{d}$. The results are presented as the $\mathrm{SNR}_{m}$ varies and for different values of the field dimension $d$. We notice that for $d=3$ our approximation is tight, for all values of $\mathrm{SNR}_{m}$, and that the approximation tightens as $\beta$ decreases.

\section{CONCLUSIONS}

We studied a wireless sensor network sampling a bandlimited, multidimensional field. We noted that, for an exact analytical study of the quality of the reconstructed field, the eigenvalue distribution of the reconstruction matrix is required. Since such a distribution is unknown, we first derived a closed-form expression of the distribution moments. By using this expression, we showed that the eigenvalue distribution of the reconstruction matrix tends to the Marčenko-Pastur distribution as the field dimension tends to infinity. We applied our results to the study of the MSE of the reconstructed field, when linear filtering is used. We found that, by using the Marčenko-Pastur distribution instead of the actual eigenvalue distribution, we obtain a close approximation to the MSE of the reconstructed signal, which is already excellent for a 3-dimensional field. We believe that our work is the basis for an analytical study of various aspects concerning the reconstruction quality of multidimensional sensor fields, and, more generally, of irregularly sampled signals.

\section{REFERENCES}

[1] H. G. Feichtinger, K. Gröchenig, and T. Strohmer, "Efficient numerical methods in non-uniform sampling theory," Numerische Mathematik, Vol. 69, pp. 423-440, 1995.

[2] A. Nordio, C.-F. Chiasserini, and E. Viterbo, "Quality of field reconstruction in sensor networks," IEEE Infocom, Anchorage, AK, May 2007.

[3] A. Nordio, C.-F. Chiasserini, and E. Viterbo, "Bandlimited field reconstruction for wireless sensor networks," http://arxiv.org/abs/0707.1954

[4] T. Strohmer, "Computationally attractive reconstruction of bandlimited images from irregular samples," IEEE Transactions on Image Processing, Vol. 6, No. 4, pp. 540-545, Apr. 1997.

[5] The on-line encyclopedia of integer sequences, http://www.research.att. $\mathrm{com} / \sim$ njas/sequences/A001263 